05

\title{
Расчет остаточных напряжений при коноскопических измерениях в профилированных монокристаллах сапфира
}

\author{
(С) С.И. Бахолдин, В.М. Крымов, Ю.Г. Носов \\ Физико-технический институт им. А.Ф. Иофрфе РАН, \\ 194021 Санкт-Петербург, Россия \\ e-mail: v.krymov@mail.ioffe.ru
}

Поступило в Редакцию 28 июля 2020 г.

В окончательной редакции 19 октября 2020 г.

Принято к публикации 20 октября 2020 г.

Рассмотрены вопросы измерения остаточных напряжений, которые образуются в профилированных монокристаллах сапфира при их выращивании из расплава. Разработан алгоритм расчета нормальных компонент тензора остаточных напряжений по данным оптических измерений остаточных напряжений методом коноскопии для оптически одноосных кристаллов в форме пластин. Приведены примеры измерения остаточных напряжений и расчета нормальных компонент для квадратного стержня и базисноограненной ленты сапфира. Показано, что нормальные напряжения сконцентрированы в основном по периферии пластин и достигают $25 \mathrm{MPa} \mathrm{в} \mathrm{стержне} \mathrm{и} 70 \mathrm{MPa} \mathrm{в} \mathrm{ленте.}$

Ключевые слова: кристаллы сапфира, остаточные напряжения, разделение компонент тензора напряжений.

DOI: $10.21883 /$ JTF.2021.04.50622.236-20

\section{Введение}

Остаточные напряжения - это напряжения, которые существуют и уравновешиваются внутри твердого тела после устранения причин их вызвавших. Это явление часто встречается в различных металлических, железобетонных и других конструкциях и деталях. Образованию остаточных напряжений, теории и методам их изучения посвящено много работ и монографий [1-3]. Развитие электроники вызвало возникновение новой отрасли: выращивание полупроводниковых, диэлектрических и других монокристаллов различных веществ. Оказалось, что при выращивании монокристаллов из расплава в них также образуются остаточные напряжения, физическая природа и механизмы образования аналогичны напряжениям в металлах [4,5]. Остаточные напряжения в кристаллах относятся к 1 роду (по классификации Н.Н. Давиденкова), поскольку они ,уравновешиваются в пределах областей, размеры которых одного порядка с размерами тела“ [1]. Механизм образования остаточных напряжений при выращивании монокристаллов из расплава близок к механизму образования остаточных напряжений в литых деталях при охлаждении отливок [1].

Для кристаллов, выращиваемых со свободной поверхности расплава и не соприкасающихся со стенками тигля, механизм образования остаточных напряжений заключается в следующем. Сложный радиационно-кондуктивный характер теплообмена в растущем кристалле приводит к значительным нелинейностям температурных полей. Они, в свою очередь, вызывают несовместность температурных деформаций и появление термоупругих напряжений. Если эти напряжения превосходят критические напряжения сдвига, то происходит пластическая деформация, и образуются дефекты структуры (дислокации), поля напряжений которых компенсируют термоупругие напряжения. После остывания кристалла термоупругие напряжения исчезают, но остаются дефекты структуры. Связанные с ними поля напряжений и есть остаточные напряжения [4-9].

Особенно актуальна проблема образования повышенных остаточных напряжений при выращивании тонкостенных изделий из расплава способом Степанова (EFG). Это относится к таким технически важным кристаллам, как сапфир [10-20], кремний [21-24], оксид галлия $[25,26]$. Профилированные монокристаллы сапфира (трубки, стержни, пластины) широко используются как конструкционные и оптические элементы приборов (окна, световоды, оболочки ламп и т.д.). Снижение уровня остаточных напряжений, остающихся в кристаллах после выращивания, очень важно для получения качественных кристаллов. При высоком уровне остаточных напряжений возможно растрескивание кристаллов при остывании или при их разрезке. Показано, что, целенаправленно изменяя конфигурацию тепловых экранов в ростовой зоне выращивания сапфировых лент, можно понижать уровень термопластических, а значит и остаточных напряжений в кристаллах [27-30].

Для разработки эффективных методов снижения остаточных напряжений очень важны способы их измерения в кристаллах различной симметрии. Наиболее часто для измерений остаточных напряжений в кристаллах некубических сингоний применяется метод коноскопии - измерение в сходящемся поляризованном свете $[6,31,32]$, позволяющий экспериментально определить в каждой точке сечения кристалла разность главных напряжений и угол изоклины, по которым можно рассчитать разность нормальных напряжений и касательное напряжение. 
В большинстве работ этими данными и ограничиваются. Но для более полной оценки распределения остаточных напряжений желательно знать распределение отдельных компонент тензора напряжений. Разделение компонент может быть выполнено несколькими способами: экспериментально - методом дополнительного наклонного просвечивания - и расчетным путем с использованием дифференциальных уравнений механики сплошной среды [33]. Первый метод неприменим для кристаллов средних сингоний, к которым относится сапфир, поскольку в них есть естественное двулучепреломление в направлениях, непараллельных оптической оси. Поэтому наиболее эффективным подходом является разделение напряжений путем численного интегрирования с использованием уравнений равновесия.

В настоящей работе разработан алгоритм такого расчета и приведены примеры измерения остаточных напряжений и последующего разделения тензора остаточных напряжений. Объектами исследования были базисноограненая лента (плоскость ленты перпендикулярна оптической оси) и пластина, вырезанная из стержня сапфира, выращенного в направлении оптической оси.

\section{1. Измерение остаточных напряжений}

Остаточные напряжения изучались методом коноскопии. Методика измерения, подробно описанная в [34], основана на том, что под действием напряжений оптически одноосный кристалл сапфира приобретает аномальную двуосность, которая проявляется в расхождении ветвей изогир коноскопической фигуры.

Коноскопические картины наблюдались с помощью поляризационного микроскопа с окуляром 10× (шкала с делениями ценой $0.1 \mathrm{~mm}$ ) и объективом $20^{\times}$. Поле зрения при этом составляло $0.7 \mathrm{~mm}$. Измерения проводили в декартовых координатах для прямоугольных образцов (рис. 1). В каждой точке сетки измеряли величину максимального расхождения изогир $2 V$ и угол $\gamma$ наклона плоскости оптических осей к оси $X$. Точность определения напряжений таким методом составляла примерно $5 \mathrm{MPa}$.

В декартовых координатах формулы для разности нормальных напряжений $\left(\sigma_{x}-\sigma_{y}\right)$ и сдвигового напряжения $\tau_{x y}$ имеют вид [34]:

$$
\begin{gathered}
\sigma_{x}-\sigma_{y}=C \cdot \operatorname{tg}^{2} V \cdot \cos 2 \gamma, \\
\tau_{x y}=\frac{C}{2} \cdot \operatorname{tg}^{2} V \cdot \sin 2 \gamma,
\end{gathered}
$$

где $C=\frac{n_{0}^{-2}-n_{e}^{-2}}{\pi_{11}-\pi_{12}}$.

Здесь $\pi_{11}$ и $\pi_{12}$ - пьезооптические коэффициенты, $n_{0}$ и $n_{e}$ - коэффициенты преломления сапфира.

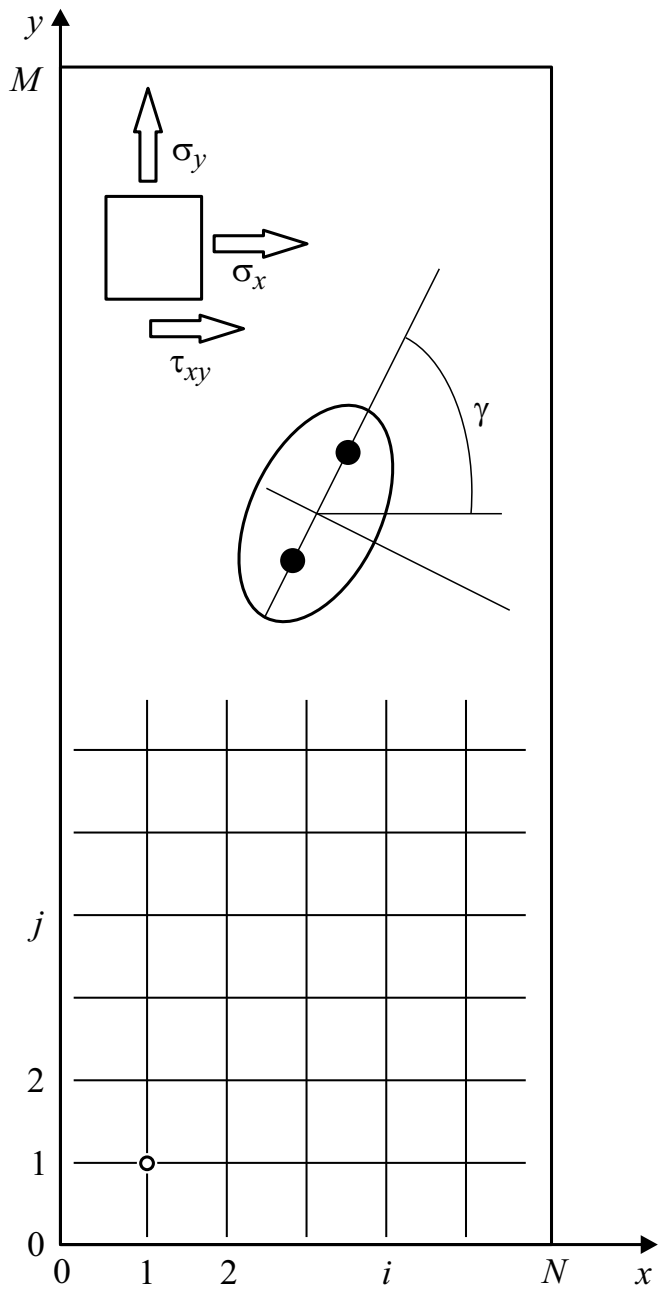

Рис. 1. Схема расчетной модели разделения напряжений в пластине. Показано сечение оптического эллипсоида плоскостью ленты. $\gamma$ - угол плоскости оптических осей с осью $x$. В нижней части пластины показана сетка для расчета напряжений методом численного интегрирования уравнений равновесия.

\section{2. Разделение напряжений}

Таким образом, измерение напряжений поляризационно-оптическим методом в кристалле в форме пластины дает два массива результатов: $\left(\sigma_{x}-\sigma_{y}\right)$ и $\tau_{x y}$. Разделение напряжений осуществлялось путем численного интегрирования уравнений равновесия:

$$
\left\{\begin{array}{l}
\frac{\partial \sigma_{x}}{\partial x}+\frac{\partial \tau_{x y}}{\partial y}=0 \\
\frac{\partial \sigma_{y}}{\partial y}+\frac{\partial \tau_{x y}}{\partial x}=0
\end{array}\right.
$$

Граничные условия: $\sigma_{x}=0$ при $x=0, x=B, \sigma_{y}=0$ при $y=0, y=L, \tau_{x y}=0$ при $x=0, x=B, y=0$, $y=L$, где $L$ и $B$ - длина и ширина пластины. Введем сетку (рис. 1): по $x: i=0,1 \ldots N$, с шагом $\Delta x$; по $y$ : $j=0,1 \ldots M$, с шагом $\Delta y$. 


\section{1. Аппроксимация нормальных напряжений оператором первого порядка}

Заменим частные производные от нормальных напряжений конечными разностями (частные производные от касательной компоненты пока трогать не будем):

$$
\begin{aligned}
& \left\{\begin{array}{l}
\frac{\sigma_{x, i, j}-\sigma_{x, i-1, j}}{\Delta x}+\left.\frac{\partial \tau_{x y}}{\partial y}\right|_{i, j}=0 \\
\frac{\sigma_{y, i, j}-\sigma_{y, i, j-1}}{\Delta y}+\left.\frac{\partial \tau_{x y}}{\partial x}\right|_{i, j}=0
\end{array}\right. \\
& \Rightarrow\left\{\begin{array}{l}
\sigma_{x, i, j}=\sigma_{x, i-1, j}-\left.\Delta x \frac{\partial \tau_{x y}}{\partial y}\right|_{i, j} \\
\sigma_{y, i, j}=\sigma_{x, i, j-1}-\left.\Delta y \frac{\partial \tau_{x y}}{\partial x}\right|_{i, j}
\end{array}\right.
\end{aligned}
$$

Граничные условия: $\sigma_{x, i, j}=0$ при $i=0, i=N, \sigma_{y, i, j}=0$ при $j=0, j=M, \tau_{x y, i, j}=0$ при $i=0, i=N, j=0$, $j=M$.

Теперь для аппроксимации $\left.\frac{\partial \tau_{x y}}{\partial x}\right|_{i, j}$ и $\left.\frac{\partial \tau_{x y}}{\partial y}\right|_{i, j}$ можно использовать любые разностные дифференциальные операторы.

\section{2. Аппроксимация касательных напряжений оператором первого порядка}

Простейший из операторов дифференцирования (с остаточным членом):

$$
y_{0}^{\prime}=\frac{1}{\Delta x}\left(-y_{-1}+y_{0}-\frac{\Delta x}{2} y^{\prime \prime}\right) .
$$

Степень аппроксимации будет такой же, как и для нормальных компонент.

$$
\left\{\begin{array}{l}
\sigma_{x, i, j}=\sigma_{x, i-1, j}-\frac{\Delta x}{\Delta y}\left(\tau_{x y, i, j}-\tau_{x y, i, j-1}\right) \\
\sigma_{y, i, j}=\sigma_{y, i, j-1}-\frac{\Delta y}{\Delta x}\left(\tau_{x y, i, j}-\tau_{x y, i-1, j}\right) .
\end{array}\right.
$$

При $\Delta x=\Delta y$ получаем расчетные соотношения:

$$
\left\{\begin{array}{l}
\sigma_{x, i, j}=\sigma_{x, i-1, j}+\tau_{x y, i, j-1}-\tau_{x y, i, j} \\
\sigma_{y, i, j}=\sigma_{y, i, j-1}+\tau_{x y, i-1, j}-\tau_{x y, i, j}
\end{array}\right.
$$

По формулам (7) нельзя вычислить $\sigma_{x}$ при $j=0$ и $\sigma_{y}$ при $i=0$ (неоткуда взять значения с индексом -1 ). На краях ленты, при $i=N$ и $j=M$, также получаются значения нормальных компонент, не соответствующие граничным условиям. Поэтому следует сразу обнулить матрицы напряжений в соответствии с граничными условиям, и вычислять недостающие значения экстраполяцией:

$$
\begin{aligned}
& \sigma_{x, i, 0}=2 \sigma_{x, i, 1}-\sigma_{x, i, 2}, \\
& \sigma_{y, 0, j}=2 \sigma_{y, 1, j}-\sigma_{y, 2, j} .
\end{aligned}
$$

Таким образом, расчет начинается с точки с координатами $i=1, \quad j=1: \quad \sigma_{x, 1,1}=\sigma_{x, 0,1}+\tau_{x y, 1,0}-$ $-\tau_{x y, 1,1}=0+\tau_{x y, 1,0}-\tau_{x y, 1,1}$, где последние два значения берутся из массива измерений $\tau_{x y}$.

\section{3. Результаты измерения и разделения напряжений}

\section{1. Тестовый пример}

Предложенный алгоритм разделения напряжений был апробирован для случая выращивания квадратного стержня. Рассмотрим упрощенную модель образования остаточных напряжений. Предположим, что в процессе выращивания кристалла максимальные термические напряжения имели место только в относительно узкой зоне вблизи фронта кристаллизации, и именно в этой области и происходила пластическая деформация, образование дефектов структуры и связанных с ними остаточных напряжений. Поскольку весь кристалл в процессе его выращивания проходил через эту область, остаточные напряжения должны быть примерно одинаковы по длине кристалла, а характер их распределения в сечении - противоположен распределению временных термоупругих напряжений. Аналитическое решение задачи расчета термоупругих напряжений в стержне прямоугольного сечения, находящегося в сложном температурном поле, было получено методом асимптотического интегрирования [35]. В качестве параметра малости используется отношение характерного поперечного размера к длине кристалла. Формулы получены для изотропного приближения, поскольку для кристаллов сапфира анизотропия теплопроводности, температурного расширения и упругих свойств невелика:

$$
\begin{aligned}
\sigma_{x} & =\alpha E T_{Z}^{\prime \prime} \frac{7}{32} \frac{1}{1+v} \frac{1}{k_{r}^{4}+\frac{4}{7} k_{r}^{2}+1}\left(x^{2}-c^{2}\right)^{2}\left(3 y^{2}-b^{2}\right), \\
\sigma_{y} & =\alpha E T_{Z}^{\prime \prime} \frac{7}{32} \frac{1}{1+v} \frac{1}{k_{r}^{4}+\frac{4}{7} k_{r}^{2}+1}\left(3 x^{2}-c^{2}\right)\left(y^{2}-b^{2}\right)^{2}, \\
\tau_{x y} & =-\alpha E T_{Z}^{\prime \prime} \frac{7}{8} \frac{1}{1+v} \frac{1}{k_{r}^{4}+\frac{4}{7} k_{r}^{2}+1} x y\left(x^{2}-c^{2}\right)\left(y^{2}-b^{2}\right),
\end{aligned}
$$

где $\alpha-$ коэффициент теплового расширения, $E-$ модуль Юнга, $v$ - коэффициент Пуассона, $k_{r}=c / b$, $2 c$ - размер в направлении оси $x, 2 b-$ в направлении оси $y$.

Конкретный уровень напряжений определяется кривизной температурного поля (значением второй производной от температуры в направлении выращивания кристалла $\left.-T_{z}^{\prime \prime}\right)$, но для оценки характера распределения можно принять $T_{z}^{\prime \prime}=1$. Для разделения напряжений достаточно рассмотреть только касательную компоненту $\tau_{x y}$, распределение которой по сечению кристалла в форме квадратного стержня будет иметь вид, показанный на рис. 2, $a$.

Применение описанного выше алгоритма разделения напряжений приводит к получению эпюр компонент $\sigma_{x}$ 

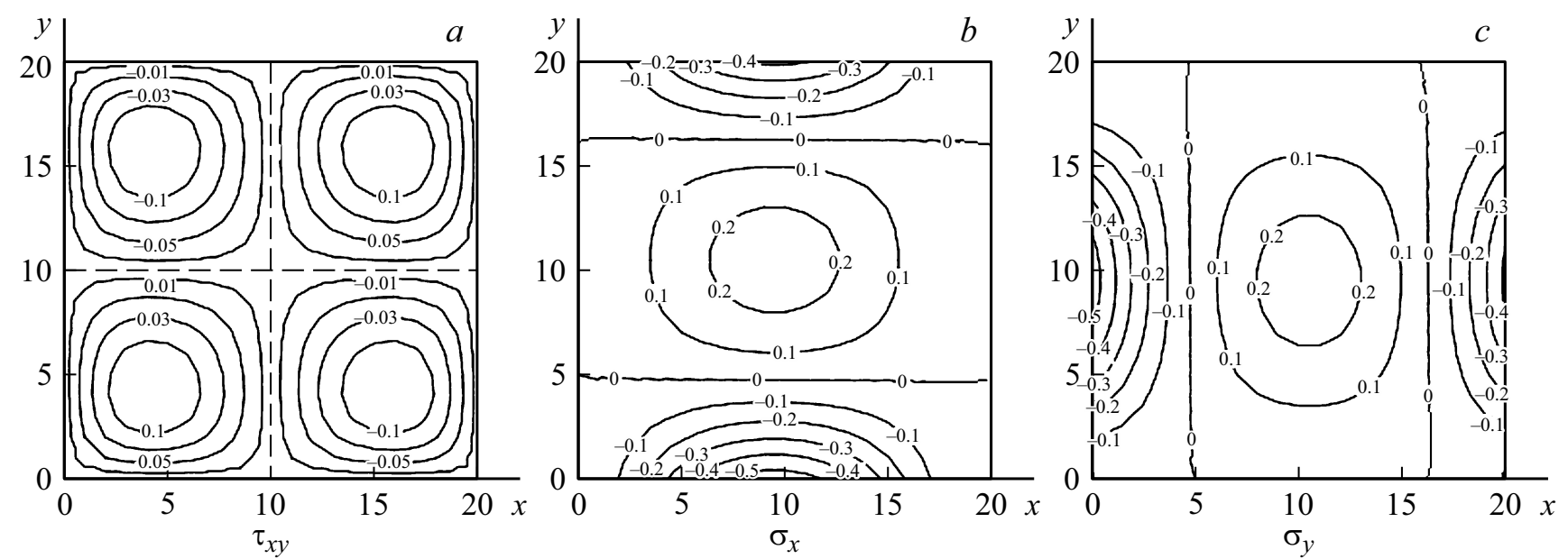

Pис. 2. Эпюры компонент термоупругих напряжений в сечении прямоугольного стержня (тестовый пример): $a-$ касательное напряжение $\tau_{x y}$, построенное по формуле $(10), b$ и $c$ - нормальные напряжения $\sigma_{x}$ и $\sigma_{y}$, полученные по алгоритму разделения напряжений (7). Значения напряжений на эпюрах — в условных единицах.

и $\sigma_{y}$, показанных на рис. $2, b$ и $c$. Видно, что напряжения концентрируются в центре и на краях пластины и имеют разный знак. Можно ожидать, что эпюры компонент остаточных напряжений по результатам измерений в прямоугольном стержне будут подобны приведенным.

\section{2. Прямоугольная пластина}

Измерения были выполнены на полированной пластинке, размером $14 \times 17 \mathrm{~mm}$ и толщиной $4 \mathrm{~mm}$, вырезанной перпендикулярно направлению выращивания [0001] из прямоугольного стержня на расстоянии $30 \mathrm{~mm}$ от затравки. Распределение аномальной двуосности по пластине измерялось на сетке с шагом $1 \mathrm{~mm}$. На рис. 3, $a$ приведены фотографии коноскопических фигур в характерных точках пластины. На рис. $3, b-$ эпюра компоненты $\tau_{x y}$, рассчитанная по формуле (2) по измеренным параметрам коноскопических фигур. На рис. 3, $c$ приведена сглаженная эпюра $\tau_{x y}$. Сглаживание экспериментальных данных проводилось по стандартной процедуре программы Mathcad (Loess - interp) с параметром $\mathrm{Span}=0.3$. После сглаживания на эпюре хорошо видны четыре области максимальных напряжений с чередующимися знаками, что соответствует рассмотренному выше тестовому примеру (рис. 2). Далее проводилось разделение напряжений по вышеописанному алгоритму (рис. 3, $d$ и $e$ ).

Видно, что нормальное напряжение $\sigma_{x}$ на краях пластины $(y=0$ и $15 \mathrm{~mm})$ и в центре пластины имеют противоположные знаки. При $x=0$ и $16 \mathrm{~mm}$ компонента $\sigma_{x}$ обращается в нуль в силу граничных условий. Качественно картина напряжений подобна модельному случаю, хотя максимум в центре выражен не столь явно. Аналогично и распределение второй нормальной компоненты $\sigma_{y}$ (рис. 3,e).
В целом уровень напряжений не превышает $25 \mathrm{MPa}$. Сопоставление измеренных остаточных напряжений по сечению прямоугольного стержня с расчетными термоупругими показывает, что их знаки противоположны, что соответствует теоретическим представлениям.

Следует отметить, что измеренные уровни напряжений в целом не влияют на оптическое пропускание и механическую прочность таких стержней. Детали, изготовленные из стержней, могут применяться в различных конструкциях, в том числе и оптических. Однако такой уровень остаточных напряжений может быть критическим при изготовлении деталей для поляризационной оптики.

\section{3. Базисноограненная сапфировая лента}

Исследованы остаточные напряжения в нижней части сапфировой ленты шириной $30 \mathrm{~mm}$ (с областью отрыва) и толщиной $1.5 \mathrm{~mm}$. Поскольку поверхности ленты были огранены с обеих сторон базисными гранями таким образом, что поверхности оказывались близки к полированным, измерения проводились без дополнительной обработки поверхностей. Измерения оптических параметров (величина расхождения изогир и ориентация плоскостей оптических осей) были проведены на сетке со сторонами $2 \times 2 \mathrm{~mm}$. По измеренным данным рассчитывались разность нормальных компонент напряжений $\left(\sigma_{x}-\sigma_{y}\right)$ и касательная компонента тензора напряжений $\tau_{x y}$. Так как величины экспериментальных значений компоненты $\tau_{x y}$ получается с большой погрешностью, проводилось сглаживание экспериментальных данных по стандартной процедуpe программы Mathcad (Loess - interp). Разделение напряжений $\sigma_{x}$ и $\sigma_{y}$ проводилось численным методом на сетке $31 \times 57$ точек по итерационным формулам (7). 
$a$
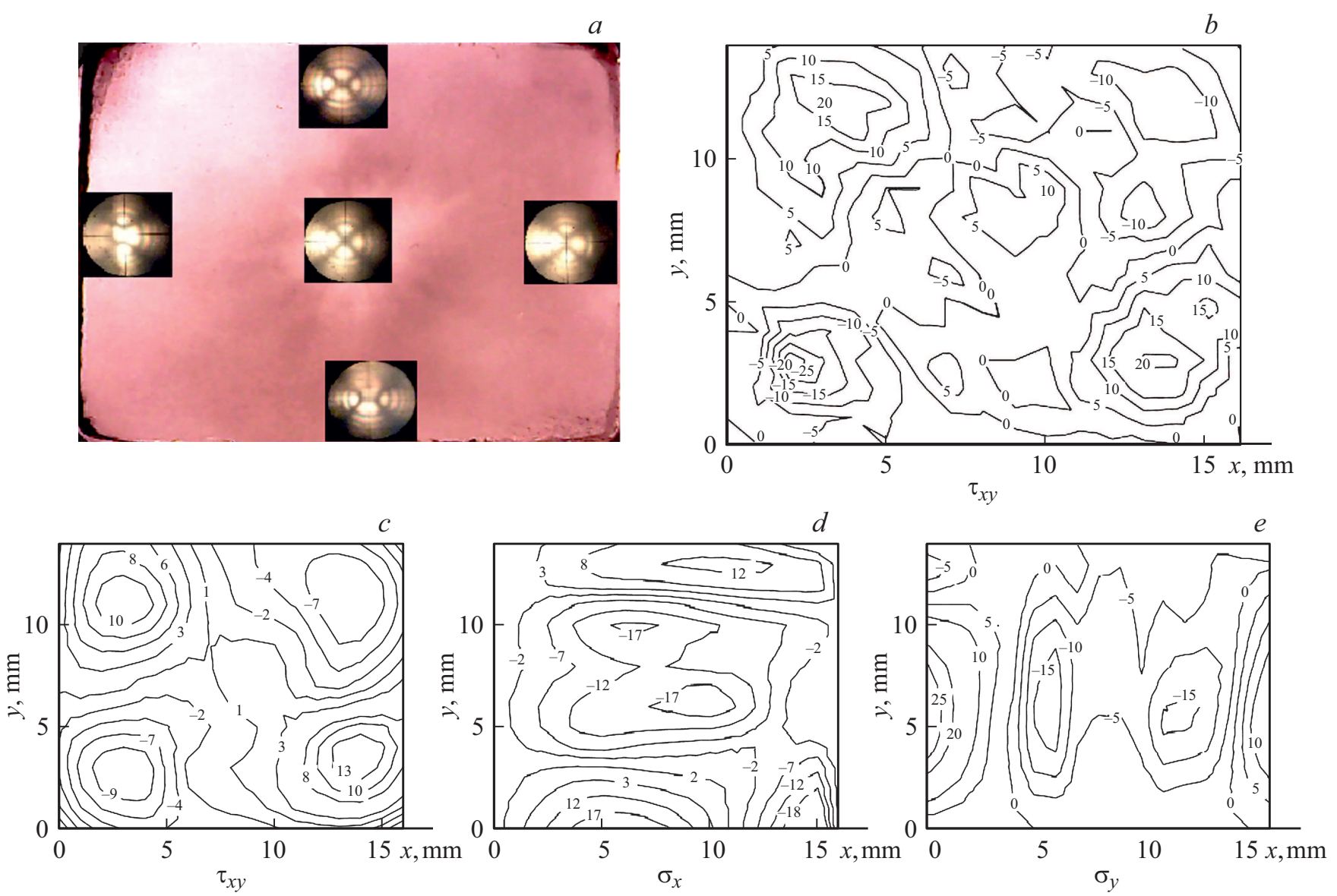

Рис. 3. Эпюры компонент остаточных напряжений в сечении прямоугольного стержня: $a-$ фотографии коноскопических фигур в различных частях пластины сапфира; $b-$ эпюра экспериментально измеренного касательного напряжения $\tau_{x y} ; c-$ сглаженная эпюра $\tau_{x y} ; d$ и $e-$ нормальные напряжения $\sigma_{x}$ и $\sigma_{y}$, полученные по алгоритму разделения напряжений (7). Значения напряжений на эпюрах — в МРa.

Для определения $\sigma_{x}$ интегрирование начинали с одной из продольных сторон ленты, а для $\sigma_{y}-$ у фронта отрыва, где эти компоненты равны нулю в силу граничных условий.

На рис. 4, $a, b$ приведены эпюры измеренных и сглаженных значений $\left(\sigma_{x}-\sigma_{y}\right)$ и $\tau_{x y}$, а на рис. $4, c, d-$ эпюры разделенных компонент тензора напряжений $\sigma_{x}$ и $\sigma_{y}$ соответственно.

Видно, что компонента тензора напряжений $\sigma_{x}$ имеет сжимающий характер, концентрируется и достигает максимальных значений на нижнем торце (до $70 \mathrm{MPa}$ ), т. е. у фронта кристаллизации при выращивании ленты. Эти остаточные напряжения по видимому связаны с дефектами, которые могли образоваться под действием термонапряжений, имевших место при выращивании в наиболее горячей зоне у фронта кристаллизации. Компонента тензора напряжений $\sigma_{y}$, направленная вдоль ленты, равна нулю на нижнем краю ленты $(y=0)$, далее при $y=15 \mathrm{~mm}$ имеет максимумы разного знака на краях и в центре ленты. На расстоянии примерно равном ширине ленты эти напряжения обращаются в нуль и растут, меняя знак. Напряжения $\sigma_{y}$ меньше по величине, чем $\sigma_{x}$. Заметим также, что в отличие от эпюр напряжений в тестовом примере в эпюрах как измеренных, так и разделенных нормальных напряжений для стержня и ленты не наблюдается полной симметрии. Это может быть связано с тем, что в реальных тепловых условиях выращивания кроме нелинейного распределения температуры вдоль оси выращивания есть и радиальные перекосы температуры. Видно, что уровень остаточных напряжений в ленте в несколько раз выше по сравнению с квадратным стержнем. Это связано с тем, что более тонкая лента в процессе роста охлаждается интенсивнее и находится в более неоднородном температурном поле по сравнению со стержнем. Опыт выращивания кристаллов сапфира показывает, что для тонких лент базисной ориентации эти напряжения могут оказаться критическими и приводить к образованию блочной структуры [11,27,32]. При других кристаллографических ориентациях (чаще всего призматических) уровень остаточных напряжения значительно меньше и изделия из них широко применяются, например, в качестве оптических элементов и часовых стекол. 

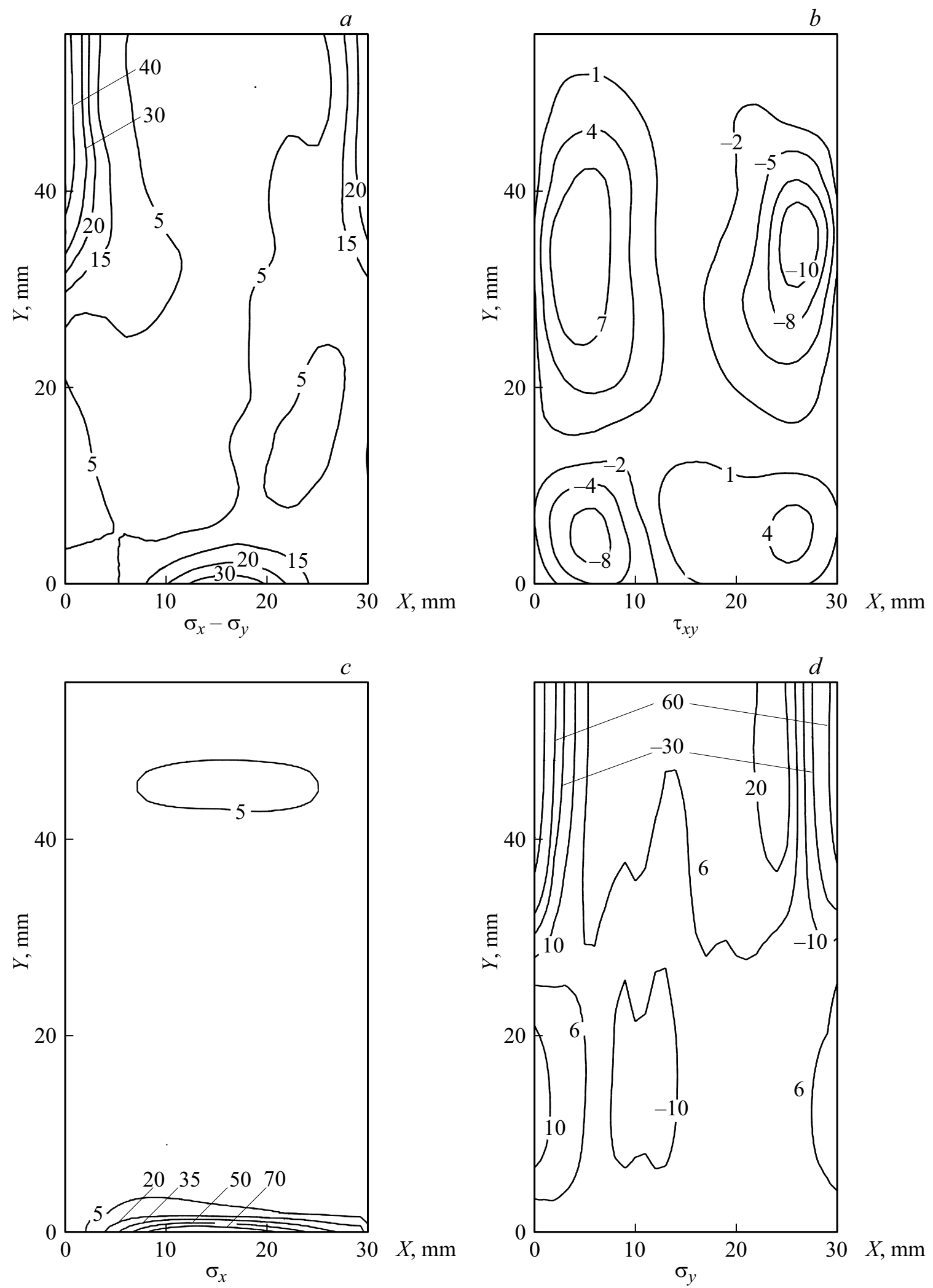

Рис. 4. Эпюры компонент остаточных напряжений в базисноограненной ленте сапфира: $a$ - эпюра разности нормальных напряжений $\sigma_{x}-\sigma_{y}$ (экспериментально измеренных и сглаженных); $b-$ то же для касательного напряжения $\tau_{x y} ; c$ и $d-$ нормальные напряжения $\sigma_{x}$ и $\sigma_{y}$, полученные по алгоритму разделения напряжений (7). Значения напряжений на эпюрах - в MРа. 


\section{Выводы}

Показано, что по данным оптических измерений коноскопических фигур и с помощью разработанного алгоритма разделения компонент тензора напряжений можно восстановить полную картину остаточных напряжений в профилированных кристаллах сапфира.

Рассмотрение полной картины компонент тензора остаточных напряжений в сечении прямоугольного стержня показывает, что по периметру сечения действуют нормальные растягивающие напряжения $\sigma_{x}$ и $\sigma_{y}$, попарно на противоположных сторонах. Максимальные значения этих напряжений достигают $25 \mathrm{MPa}$. В центре сечения действуют сжимающие $\sigma_{x}$ и $\sigma_{y}$, (до $17 \mathrm{MPa}$ ). В лентах максимальные сжимающие напряжения $\sigma_{x}$ концентрируются на нижнем торце ленты, и они во многом формируют дислокационную и блочную структуру лент.

Полученные результаты в сочетании с расчетами полей термоупругих напряжений, которые можно выполнить на основе измерения температурного поля в выращиваемых кристаллах, позволяют целенаправленно управлять уровнем остаточных напряжений в кристаллах.

\section{Благодарности}

Авторы выражают благодарность А.В. Денисову за измерения остаточных напряжений в сапфировой ленте.

\section{Конфликт интересов}

Авторы заявляют, что у них нет конфликта интересов.

\section{Список литературы}

[1] Я.Б. Фридман. Механические свойства металлов (Машиностроение, М., 1974)

[2] И.А. Биргер. Остаточные напряжсения (Машгиз, М., 1963)

[3] А.А. Поздеев, Ю.И. Няшин, П.В. Трусов. Остаточные напряжения: теория и приложения (Наука, М., 1982)

[4] А.А. Чернов, Е.И. Гиваргизов, Х.С. Багдасаров, В.А. Кузнецов, Л.Н. Демьянец, А.Н. Лобачев. Современная кристаллография, T. 3 (Наука, М., 1980)

[5] В.Л. Инденбом. Кристаллография, 9 (1), 74 (1964).

[6] А.Г. Штукенберг, Ю.О. Пунин. Оптические аномалии в кристаллах (Наука, СПб., 2004)

[7] E.R. Dobrovinskaya, L.A. Litvinov, V. Pischik. Sapphire: Material, Manufacturing, Applications (Springer-Verlag, Berlin, 2009). DOI: 10.1007/978-0-387-85695-7

[8] P.J. Withers. Rep. Prog. Phys., 70, 2211 (2007). DOI: 10.1088/0034-4885/70/12/R04

[9] C.K. Bhihe, P.A. Mataga, J.W. Hutchinson, S. Rajendran, J.P. Kalejs. J. Cryst. Growth, $137(1-2), 86$ (1994). DOI: 10.1016/0022-0248(94)91252-1

[10] V.M. Krymov, Yu.G. Nosov, S.I. Bakholdin, V.N. Maslov, I.L. Shul' pina, V.I. Nikolaev. J. Cryst. Growth, 457, 314 (2017). DOI: org/10.1016/j.jcrysgro.2016.08.017
[11] E.F. Dolzhenkova, A.V. Voloshin, L.A. Lytvynov, R.I. Safronov. Cryst. Res. Technol., 53 (2), 1700258 (2018). DOI: $10.1002 /$ crat. 201700258

[12] А.Е. Андреев, Е.Ф. Долженкова, П.В. Коневский, Л.А. Литвинов, О.А. Лукиенко. Неорган. матер., 51 (10), 1105 (2015). DOI: 10.7868/S0002337X15100012 [A.E. Andreev, E.F. Dolzhenkova, P.V. Konevskii, L.A. Litvinov, O.A. Lukienko. Inorganic Mater., 51 (10), 1025 (2015). DOI: 10.1134/S0020168515100015]

[13] F. Theodore, T. Duffar, J.L. Santailler, J. Pesenti, M. Keller, P. Dusserre, F. Louchet, V. Kurlov. J. Cryst. Growth, 204, 317 (1999). DOI: org/10.1016/S0022-0248(99)00157-8

[14] В.А. Бородин, А.В. Жданов, М.В. Юдин. Изв. РАН Сер. физ., 68 (6), 825 (2004). [V.A. Borodin, A.V. Zhdanov, M.V. Yudin. Bull. Russ. Acad. Sci. Phys., 68 (6), 933 (2004).]

[15] A.V. Borodin, V.A. Borodin, D.N. Frantsev, M.V. Yudin, T.A. Mosharov. J. Crystal Growth, 275, e2105 (2005). DOI: $10.1016 /$ j.jcrysgro.2004.11.295

[16] А.В. Жданов, А.В. Бородин, М.В. Юдин. Изв. РАН, Сер. физ., 73 (10), 1407 (2009). [A.V. Zhdanov, A.V. Borodin, M.V. Yudin, Bull. Russ. Acad. Sci. Phys., 73(10), 1328 (2009). DOI: 10.3103/S1062873809100050]

[17] Yu. Qinghua, Liu. Lijun, Geng A’nan, Jiang Biwen, Li Zaoyang, Xu Yangyi, Xue Kangmei. J. Cryst. Growth, 385, 49 (2014). DOI: 10.1016/j.jcrysgro.2013.01.054

[18] С.В. Гавриш, В.В. Логинов, С.В. Пучнина. Успехи прикладной физики, 6 (4), 333 (2018).

[19] С.В. Гавриш, В.В. Логинов, Д.Ю. Пугачев, С.В. Пучнина. Успехи прикладной физики, 7 (5), 480 (2019).

[20] В.М. Крымов, Ю.Г. Носов, С.И. Бахолдин, В.Н. Маслов, И.Л. Шульпина, М.П. Щеглов. ФТТ, 57 (11), 2190 (2015). DOI: org/10.1134/S1063783415110207. [V.M. Krymov, Yu.G. Nosov, S.I. Bakholdin, V.N. Maslov, I.L. Shul'pina, M.P. Shcheglov. Phys. Solid State, 57 (11), 2257 (2015). DOI: $10.1134 / \mathrm{S} 1063783415110207]$

[21] M.C. Brito, Alves J. Maia, J.M. Serra, R.M. Gamboa, C. Pinto, A.M. Vallera. Solar Energy Mater. Solar Cells, 87, 311 (2005).

[22] V. Ganapativ, S. Schoenfelder, S. Castellanos, A. Sampson, T. Buonassisi, R. Koepge, J. Bagdahn, S. Oener, H. Morhenn, G. Hahn, M.A. Marcus, B. Lai. J. Appl. Phys., 108 (6), 063528-13 (2010). DOI: 10.1063/1.3468404

[23] R.W. Gurtler, A. Baghdadi, R.J. Ellis, I.A. Lesk. J. Electron. Mater., 7 (3), 441 (1978).

[24] R.W. Gurtler. J. Cryst. Growth, 50, 69 (1980). DOI: $10.1016 / 0022-0248(80) 90229-8$

[25] A. Kuramata, K. Koshi, S. Watanabe, Yu. Yamaoka, T. Masui, S. Yamakoshi. Jpn. J. Appl. Phys., 55, 1202A2 (1-6) (2016).

[26] L. Chengcheng, L. Zaoyang, M. Wenxiang, J. Zhitai, L. Lijun. J. Cryst. Growth, 506, 83 (2019).

DOI: org/10.1016/j.jcrysgro.2018.10.029

[27] В.М. Крымов, А.В. Денисов, М.И. Саллум, С.И. Бахолдин, В.М. Мамедов, В.С. Юферев, А.А. Русанов, П.В. Смирнов. Изв. РАН. Сер. физ., 73 (10), 1436 (2009). [V.M. Krymov, A.V. Denisov, M.I. Sallum, S.I. Bakholdin, V.M. Mamedov, V.S. Yuferev, A.A. Rusanov, P.V. Smirnov. Bull. Russ. Acad. Sci. Phys., 73 (10), 1355 (2009). DOI: $10.3103 / \mathrm{S} 1062873809100116]$

[28] A.V. Denisov, A. Molchanov, Yu.O. Punin, V.M. Krymov, G. Muller, J. Friedrich. J. Cryst. Growth, 344, 38 (2012). DOI: $10.1016 /$ j.jcrysgro.2012.02.005

[29] L. Montalto, N. Paone, L. Scalise, D. Rinaldi. Rev. Sci. Instrum., 86, 063102 (2015). DOI: .org/10.1063/1.4921870] 
[30] L. Montalto, P. Natali, L. Scalise, N. Paone, F. Davì, D. Rinaldi, G. Barucca, P. Mengucci. Crystals, 9, 376 (2019). DOI: $10.3390 /$ cryst9070376

[31] В.Л. Инденбом, Г.Е. Томиловский. Кристаллография, 3, 594 (1958).

[32] А.В. Денисов, Ю.О. Пунин, А.Г. Штукенберг, О.С. Грунский. Вестник СПбГУ, Сер. 7 (1), 106 (2006).

[33] А.Я. Александров, М.Х. Ахметзянов. Поляризационно-оптические методы механики дебормируемого тела (Наука, М., 1973).

[34] А.В. Денисов, В.М. Крымов, Ю.О. Пунин. ФТТ, 49 (3), 454 (2007). [A.V. Denisov, V.M. Krymov, Yu.O. Punin. Phys. Solid State, 49, 472 (2007). DOI: 10.1134/S1063783407030146]

[35] С.И. Бахолдин, Е.В. Галактионов, В.М. Крымов. В сб.: Вопросы математической бизики и прикладной математики, под ред. Э.А. Троппа (ФТИ им. Иоффе РАН, СПб., 2011), с. 209. 